\title{
Atomic-Scale Mechanisms for Interfacial Radiation Damage Resistance of Thin Film Oxide Heterostructures
}

Steven R. Spurgeon ${ }^{1}$, Michel Sassi ${ }^{2}$, Tiffany Kaspar ${ }^{2}$, Weilin Jiang ${ }^{1}$ and Vaithiyalingam Shutthanandan ${ }^{3}$

1. Energy and Environment Directorate, Pacific Northwest National Laboratory, Richland, USA

2. Physical and Computational Sciences Directorate, Pacific Northwest National Laboratory, Richland, USA

3. Environmental Molecular Sciences Laboratory, Pacific Northwest National Laboratory, Richland, USA

Functional oxide interfaces have long been employed in the electronics, sensors, and energy storage devices central to modern life. Extensive past study has revealed a subtle interplay of structure, chemistry, and composition that govern the properties of these interfaces, unlocking ways to engineer useful properties such interfacial magnetism and ferroelectricity [1]. However, far less attention has been paid to the response of these systems in extreme environments, such as the high radiation fields experienced in nuclear reactor cores and space flight. Oxide-based devices are central components of mission-critical in-core sensors and spacecraft avionics systems. A predictive understanding of device performance and lifetimes therefore depends on better understanding and control of the radiation damage response of these materials.

Over the past decade, we have developed a comprehensive approach to engineer the properties of functional oxide heterostructures [2]. We utilize precision synthesis methods, such as molecular beam epitaxy and pulsed laser deposition, coupled with aberration-corrected scanning transmission electron microscopy (STEM) to interrogate atomic-scale structure, chemistry, and composition. Ab initio modeling serves as a bridge between synthesis and characterization, allowing us to explore inaccessible spatiotemporal regimes and providing insight into underlying structure-property relationships. Here we employ this approach to examine the radiation damage response of model perovskite-type heterostructures. While grain boundaries in metals are known to respond much differently than the bulk, radiation damage accommodation by oxide interfaces is less well understood, necessitating the use of finely controlled model systems.

We present an analysis of heteroepitaxial $\mathrm{La}_{2} \mathrm{Ti}_{2-\mathrm{x}} \mathrm{Zr}_{\mathrm{x}} \mathrm{O}_{7} / \mathrm{SrTiO}_{3}$ and related systems that have been irradiated to a range of doses using $1 \mathrm{MeV} \mathrm{Zr}{ }^{+}$ions to simulate the effect of fission fragment damage. We examine the onset of radiation damage using aberration-corrected STEM, energy-dispersive X-ray spectroscopy (EDS), and electron energy loss spectroscopy (EELS). As shown in Figure 1, we find that the interface exhibits markedly improved damage resistance, in contrast to the bulk of the film or substrate. Molecular dynamics simulations shown in Figure 2 show that the accommodation of electronic-type damage (comprising half the total damage at this energy) is directly connected to the bandgap of the crystal, as well as the ability of the octahedral network to accommodate distortions [3]. Using EELS, we examine the local electronic fine structure of the interface and identify the salient features mediating the enhanced damage response. Our study indicates that the engineering of oxide interfaces may offer a means to tune both functional properties and damage resistance, with potential implications for device design in extreme environments. 


\section{References:}

[1] Spurgeon, S. R. et al. Polarization screening-induced magnetic phase gradients at complex oxide interfaces. Nat. Commun. 6, 6735 (2015).

[2] Spurgeon, S. R. \& Chambers, S. A. Atomic-Scale Characterization of Oxide Interfaces and Superlattices Using Scanning Transmission Electron Microscopy. in Reference Module in Chemistry, Molecular Sciences and Chemical Engineering 1-11 (Elsevier, 2017).

[3] Sassi, M., Kaspar, T., Rosso, K. M. \& Spurgeon, S. R. Effect of structure and composition on the electronic excitation induced amorphization of $\mathrm{La}_{2} \mathrm{Ti}_{2-\mathrm{x}} \mathrm{Zr}_{\mathrm{x}} \mathrm{O}_{7}$ ceramics. (2018). Arxiv: 1812.01136
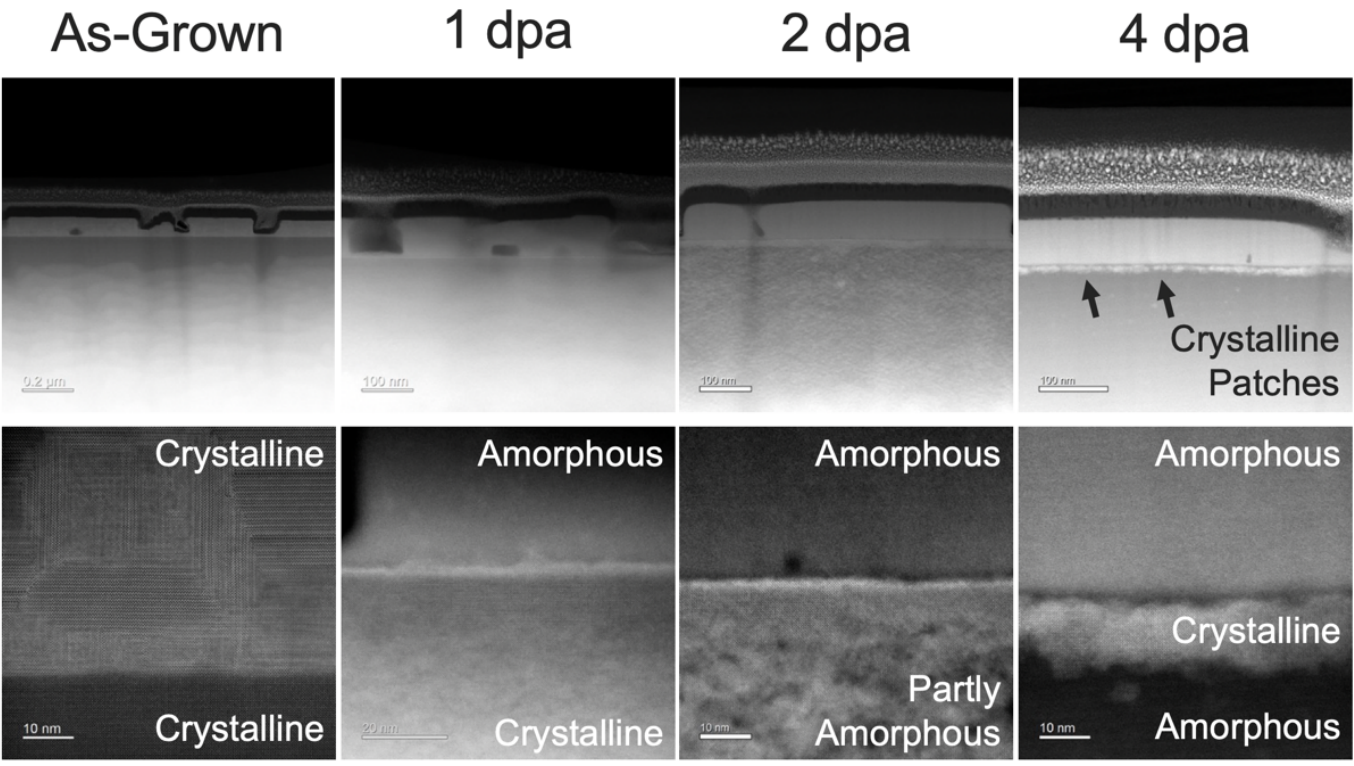

Figure 1. Cross-sectional high-angle annular dark field (STEM-HAADF) images showing the progression of amorphization with increasing dose from $0-4 \mathrm{dpa}$, as well as the preservation of a crystalline band at the film-substrate interface.

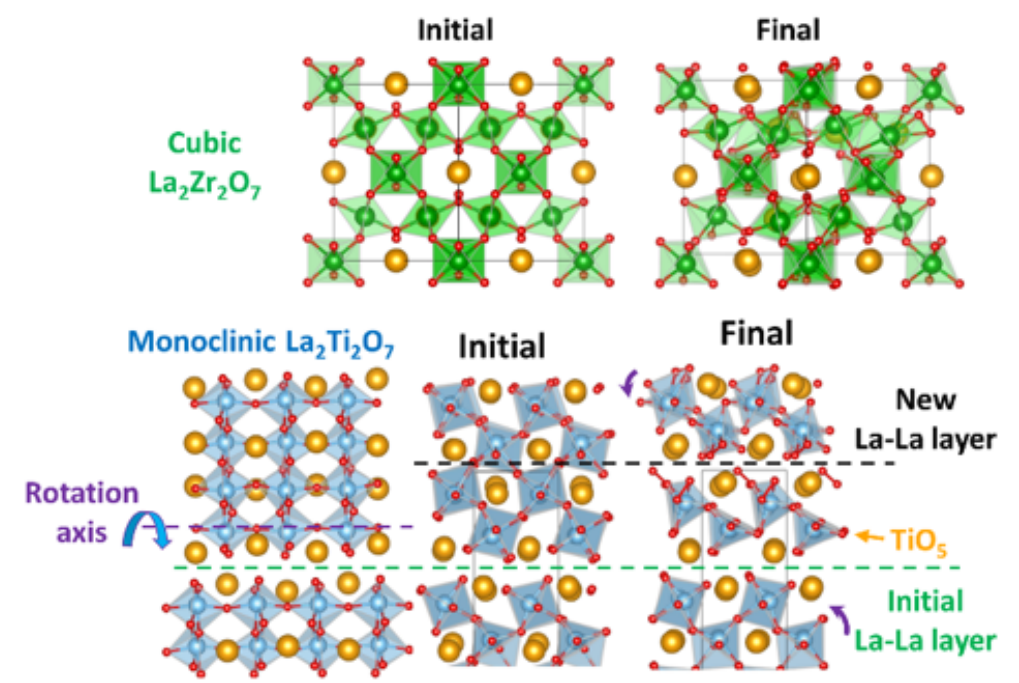

Figure 2. Molecular dynamics simulation of the accommodation of radiation damage via the distortion of the octahedral network. 